\title{
Efecto del estrés salino sobre la morfología y fitoquímica de orégano mexicano (Lippia graveolens Kunth) cultivado in vitro
}

\author{
Effect of saline stress on the morphology and phytochemistry of in vitro grown mexican oregano \\ (Lippia graveolens Kunth)
}

\begin{abstract}
Rayn Clarenc Aarland, Osvaldo Adrián Castellanos-Hernández, Araceli Rodríguez-Sahagún, Gustavo Javier AcevedoHernández*

Laboratorio de Biología Molecular Vegetal, Centro Universitario de la Ciénega, Universidad de Guadalajara. Avenida Universidad, No.1115, Col. Lindavista, CP. 47810, Ocotlán, Jalisco. México.
\end{abstract}

\section{RESUMEN}

Las plantas, al ser organismos sésiles sufren diferentes tipos de estreses bióticos y abióticos. En este trabajo se cultivaron in vitro plantas de orégano, bajo condiciones de estrés salino ( $\mathrm{NaCl} 25 \mathrm{mM})$, en diferentes fuentes de luz: blanca (CTL), ultravioleta tipo C (UV-C) y de amplio espectro (AE). Se evaluaron cambios morfológicos en las plantas tratadas, así como parámetros fitoquímicos (fenoles y flavonoides totales y la capacidad antioxidante). Los tratamientos $\mathrm{NaCl} / \mathrm{CTL}$ y $\mathrm{AE}$ mostraron el mayor número de yemas activadas. La luz UV-C mostró la menor cantidad de yemas y de altura de planta, sin embargo, no se observó efecto de la salinidad. La luz UV-C causó el menor número de hojas, así como raíces adventicias. No parece haber injerencia de la condición salinidad en estos resultados. La condición que provocó el mayor contenido de fenoles fue la luz UV-C y la que provocó el mayor contenido de flavonoides fue la combinación NaCl/UV-C. La combinación $\mathrm{NaCl} / \mathrm{AE}$ causó la mayor capacidad antioxidante con el método DPPH y ABTS. No se observó una correlación entre el contenido de fenoles y la capacidad antioxidante.

Palabras clave: Lippia graveolens, estrés, fenoles totales, capacidad antioxidante

\section{ABSTRACT}

Plants, being sessile organisms, suffer from a range of biotic and abiotic stress. In this investigation, oregano plants were grown in vitro under saline stress conditions $(\mathrm{NaCl} 25$ $\mathrm{mM})$, combined with different types of lights: white light $(C T L)$, ultraviolet- $C$ (UV-C) and broad-spectrum light (AE). Morphological changes were evaluated in the treated plants, as well as phytochemical parameters (total phenolic compounds, total flavonoids and antioxidant capacity). NaCl/CTL and $\mathrm{AE}$ conditions showed the highest numbers of activated buds. UV-C light showed the lowest number of buds and plant height, however, no effect from salinity was observed. Also, UV-C light caused the lowest amount of leaves and adventitious roots, which do not seem to be influenced by salinity. The stress condition that caused the highest amount of phenols was UV-C light, while the combination of $\mathrm{NaCl}$ / UV-C showed the highest number of total flavonoids. As for the antioxidant capacity, $\mathrm{NaCl} / \mathrm{AE}$ showed the highest capacity using both the DPPH and ABTS method. No correlation between phenols and antioxidant capacity was observed. Keywords: Lippia graveolens, stress, total phenols, antioxidant capacity

\section{INTRODUCCIÓN}

La familia Verbenaceae comprende alrededor de 35 géneros y un aproximado de 1000 especies que se distribuyen en regiones áridas y semiáridas del mundo, mayoritariamente del continente americano (Rivera et al., 2010; Herrera-Rodríguez et al, 2019). En México se conocen 26 géneros y alrededor de 286 especies (López-Villafranco et al., 2017). Una de las plantas mexicanas pertenecientes a esta familia es Lippia graveolens Kunth (Orégano mexicano). Esta planta tiene usos culinarios, en la farmacéutica, así como para tratamiento de diversos padecimientos (Pascual et al., 2001; González-Trujano et al., 2017), entre otros. Por la zona donde crece el orégano naturalmente, éste tiene que soportar periodos de poca precipitación lo cual puede resultar en situaciones de estrés (Mata-González y Meléndez-González, 2005; Martínez-Hernández et al., 2015).

Las plantas, al ser organismos sésiles, están expuestas todo el tiempo a diferentes tipos de estrés biótico y abiótico. Uno de los estreses abióticos más importantes es el estrés salino, y éste puede llegar a afectar de manera negativa su crecimiento y desarrollo (Keyvan, 2010; Rahdari y Hoseini, 2012; Zlatev y Lidon, 2012; Rana et al., 2013). En general, los síntomas de estrés salino pueden ser la pérdida de turgencia en las hojas, marchitez, amarillamiento, y la caída de las hojas (Kotagiri y Kolluru, 2017). Además, hay una acumulación de especies reactivas de oxígeno (Sharma, 2019). Sin embargo, las plantas con el paso del tiempo han desarrollado mecanismos para poder soportar los cambios ambientales y de esa manera no afectar sus procesos fisiológicos (Isah, 2019).

Los efectos del estrés salino afectan la productividad de distintos cultivos. Mundialmente, casi el $20 \%$ de las áreas cultivadas y alrededor del $30 \%$ de las áreas irrigadas del mundo se encuentra en condiciones de estrés salino y esta cifra va en aumento con una velocidad de $10 \%$ por año. El riego con agua subterránea altamente salada, la liberación de agua de mar en las zonas costeras y la acumulación de sales en regiones áridas son las principales causas de la salinidad de los suelos. Los efectos adversos en el crecimiento 
de las plantas en suelos salinos incluyen afectaciones en el crecimiento y desarrollo de las plantas, compactación de los suelos, estrés osmótico, deficiencias nutricionales y toxicidad por iones como $\mathrm{Na}^{+}$y $\mathrm{Cl}^{-}$(Isayenkov y Maathuis, 2019; Razzaq et al, 2019; Sharif et al., 2019). Adicionalmente, la salinidad suele ir acompañada por estrés oxidante debido a la sobreproducción de especies reactivas de oxígeno (ERO) (Isayenkov y Maathuis, 2019). Estas ERO interfieren con el metabolismo normal de las células y pueden causar daño, oxidando a proteínas, lípidos, ADN y otras macromoléculas (Gill y Tuteja, 2010; Sarker et al., 2018). El objetivo de este trabajo fue determinar el efecto del estrés salino en diferentes condiciones de luz, sobre la morfología y fitoquímica de plántulas de orégano mexicano cultivadas en condiciones in vitro.

\section{MATERIALES Y MÉTODOS \\ Diseño del experimento}

En este estudio se trabajó con un diseño multifactorial en el que se estudió el efecto del cloruro de sodio $25 \mathrm{mM}$ $(\mathrm{NaCl})$ en condiciones de luz blanca $(\mathrm{CTL})$, con luz de amplio espectro (AE) o luz ultravioleta tipo C (UV-C) en plantas de orégano mexicano, cultivadas in vitro (Tabla 1).

\begin{tabular}{|c|c|c|c|}
\hline $\mathrm{NaCl}(\mathrm{mM})$ & & Luces & \\
\hline & Blanca & $A E$ & UV-C \\
\hline 0 & CTL & $A E$ & UV-C \\
\hline 25 & $\mathrm{NaCl} / \mathrm{CTL}$ & $\mathrm{NaCl} / \mathrm{AE}$ & $\mathrm{NaCl} / \mathrm{UV}-\mathrm{C}$ \\
\hline
\end{tabular}

CTL luz blanca (control); $A E$, luz de amplio espectro; UV-C, luz Ultravioleta tipo $C$

\section{Material biológico}

Se germinaron semillas de Lippia graveolens Kunt provenientes de plantas silvestres, colectadas en el municipio de Colotlán Jalisco en Octubre del 2017. A partir de estas plantas se obtuvo material para el establecimiento in vitro.

\section{Reactivos químicos}

Todos los reactivos fueron adquiridos de SigmaAldrich, St. Louis, MO.

\section{Establecimiento del cultivo in vitro}

Se establecieron yemas axilares de L. graveolens Kunth provenientes de plantas de 90 días en medio MS (Murashige y Skoog, 1962), suplementado con sacarosa $30 \mathrm{~g} \mathrm{~L}^{-1}$ y phytagel $3.5 \mathrm{~g} \mathrm{~L}^{-1}$. Para el estrés salino, se suplementó con $\mathrm{NaCl} 25 \mathrm{mM}$. El pH del medio de cultivo se ajustó a 5.8, usando $\mathrm{NaOH} 0.1$ $\mathrm{M}$. Posteriormente, las plantas fueron sometidas a diferentes condiciones de luz CTL, AE y UV-C (fotoperiodo 16/8 h). Para la luz UV-C fueron pulsaciones de 2 horas diarias durante 2 meses. Posteriormente, se mantuvieron bajo condiciones de luz blanca para los análisis. Se trabajó con 3 réplicas por cada frasco y tres frascos por tratamiento (9 unidades experimentales).

\section{Evaluación de características morfológicas}

Las características morfológicas que se evaluaron fueron número de yemas activadas, altura de la planta (parte aérea), el número de hojas y número de raíces adventicias.

\section{Preparación de extractos metanólicos}

Se pesaron 200 mg de partes aéreas secas de las plántulas de orégano, se pulverizó en un mortero y se extrajo con un equipo Soxhlet con $100 \mathrm{~mL}$ de metanol durante 3 horas. Los extractos obtenidos fueron filtrados, concentrados por rota-evaporación a presión reducida hasta un volumen de 25 $\mathrm{mL}$ (IKA ${ }^{\circ}$ Staufen, Alemania) y posteriormente almacenados a $4{ }^{\circ} \mathrm{C}$ hasta su uso. Estos $25 \mathrm{~mL}$ fueron usados como extracto crudo.

\section{Determinación de compuestos fenólicos totales}

El contenido de compuestos fenólicos totales se determinó usando el reactivo de Folin-Ciocalteu mediante la técnica descrita por Singleton y Rossi (1965). Se mezcló una alícuota de $200 \mu \mathrm{L}$ de cada extracto crudo de orégano con 1 $\mathrm{mL}$ del reactivo de Folin-Ciocalteu (previamente diluido con agua en proporción 1:10 v/v) y se incubó durante 1 min a temperatura ambiente antes de adicionarle $0.8 \mathrm{~mL}$ de carbonato de sodio $(7.5 \% \mathrm{p} / \mathrm{v})$. La mezcla de reacción se incubó durante $1 \mathrm{~h}$ a temperatura ambiente y posteriormente se determinó la absorbancia a $765 \mathrm{~nm}$. El contenido total de compuestos fenólicos se reportó como equivalentes de ácido gálico (EAG) en $1 \mathrm{~g}$ de peso seco (gps) de orégano.

\section{Determinación de flavonoides totales}

Para la determinación de los flavonoides totales, se utilizó el método colorimétrico de cloruro de aluminio (Chang et al., 2002). $0.5 \mathrm{~mL}$ de los extractos crudos de orégano se mezclaron con $1.5 \mathrm{~mL}$ de metanol, $0.1 \mathrm{~mL}$ de cloruro de aluminio al $10 \%(\mathrm{p} / \mathrm{v}), 0.1 \mathrm{~mL}$ de acetato de potasio $1 \mathrm{M} \mathrm{y}$ $2.8 \mathrm{~mL}$ de agua destilada. La mezcla se incubó a temperatura ambiente durante 30 minutos y se determinó la absorbancia a $415 \mathrm{~nm}$. Se preparó una curva de calibración de quercetina de $10-100 \mu \mathrm{g} / \mathrm{mL}$ en metanol. Los resultados se reportaron en $\mathrm{mg}$ de quercetina por $\mathrm{g}$ de peso seco de orégano.

\section{Determinación de la capacidad antioxidante por DPPH}

Para la determinación de la capacidad antioxidante se preparó una solución de 2,2-difenil-2-picril-hidracil (DPPH) $0.1 \mathrm{mM}$ en metanol. Se preparó una solución diaria mezclando $10 \mathrm{~mL}$ de esta solución $0.1 \mathrm{mM}$ con $40 \mathrm{~mL}$ de metanol. La mezcla de reacción contenía $50 \mu \mathrm{L}$ de extracto crudo de orégano y $950 \mu \mathrm{L}$ de la solución diaria de DPPH (Brand Williams et al., 1995). La curva patrón fue preparada con ácido 6-hidroxi-2,5,7,8 tetrametilcroman 2-carboxílico (trolox).

\section{Determinación de la capacidad antioxidante por ABTS}

El método ABTS se realizó de acuerdo con lo descrito por Re et al. (1999) con ligeras modificaciones. Se preparó una solución de ácido2,2-azino-bis-(3-etilbenzotiazolina)-6sulfónico (ABTS) $7 \mathrm{mM}$ con persulfato de potasio $2.45 \mathrm{mM}$ en $100 \mathrm{~mL}$ de agua destilada y se dejó reaccionar durante 16 
horas. Posteriormente, se ajustó el pH de esta solución a 7.4 con amortiguador PBS $1 \mathrm{X}$ y se ajustó la absorbancia a 0.7. La mezcla de reacción constaba de una alícuota de $100 \mu \mathrm{L}$ de la respectiva dilución de cada extracto crudo de Lippia y 1 $\mathrm{mL}$ de ABTS y se determinó a $734 \mathrm{~nm}$. Los resultados para DPPH y ABTS se expresaron como capacidad antioxidante equivalentes de trolox (CAET) en $\mathrm{mM} / \mathrm{gramos}$ de peso seco.

\section{Análisis estadístico}

Todos los análisis se llevaron a cabo por triplicado. A los valores obtenidos de fenoles y flavonoides totales, DPPH y ABTS se les aplicó estadística descriptiva (media y desviación estándar), los datos morfológicos fueron analizados mediante análisis de varianza (ANOVA) de una vía y comparación múltiple de medias de Tukey $(p \leq 0.05)$.

\section{RESULTADOS Y DISCUSIÓN Morfología}

La respuesta de las plantas a altas concentraciones de sal se puede dividir en dos etapas. En la primera, que generalmente empieza en minutos o días después de las condiciones estresantes, se observa una reducción del crecimiento, cierre de estomas e inhibición de la expansión celular. En la segunda etapa que empieza en días o semanas hay una acumulación de iones, teniendo como consecuencia una desaceleración de procesos metabólicos (Isayenkov y Maathuis, 2019). Los tratamientos $\mathrm{NaCl} / \mathrm{CTL}$ y NaCl/AE mostraron el mayor número de yemas activadas. Los tratamientos UV-C y NaCl/UV-C mostraron la menor cantidad de yemas (Figura 1). Las plantas en las que se observó una mayor longitud de la parte aérea fueron aquellas sometidas a tratamientos $A E$ seguido por $\mathrm{NaCl} / \mathrm{AE}$. Las plantas tratadas con luz ultravioleta (UV-C y NaCl/UV-C) fueron las que menos crecieron (Figura 2). En cuanto al número de hojas, las plantas del tratamiento $\mathrm{NaCl} / \mathrm{AE}$ mostraron la mayor respuesta y las de la combinación $\mathrm{NaCl} / \mathrm{UV}-\mathrm{C}$ el menor número de hojas por explante (Figura 3). Varios autores han reportado que el estrés salino afecta el crecimiento y desarrollo de las plantas, debido a una acumulación de iones que causa un desbalance iónico celular (Isayenkov y Maathuis, 2019; Razzaq et al, 2019; Sharif et al., 2019). Sin embargo, en nuestro estudio se observó que la condición que más afectó el desarrollo de las plantas fue la luz UV-C. En cuanto a número de raíces adventicias, el mejor tratamiento fue $\mathrm{NaCl} / \mathrm{CTL}$ y la que mostró menor número de raíces adventicias de nuevo fueron los tratamientos con UV-C (Figura 4). Además, se ha visto en otras plantas que el estrés salino causa un incremento en el número de raíces adventicias (Arif et al., 2019), como se pudo observar en nuestro estudio.

\section{Compuestos fenólicos totales}

En las plantas superiores, el carbono que no es utilizado para el crecimiento y desarrollo puede ser redirigido para la síntesis de compuestos de almacenamiento como el almidón o de metabolitos secundarios basados en carbono

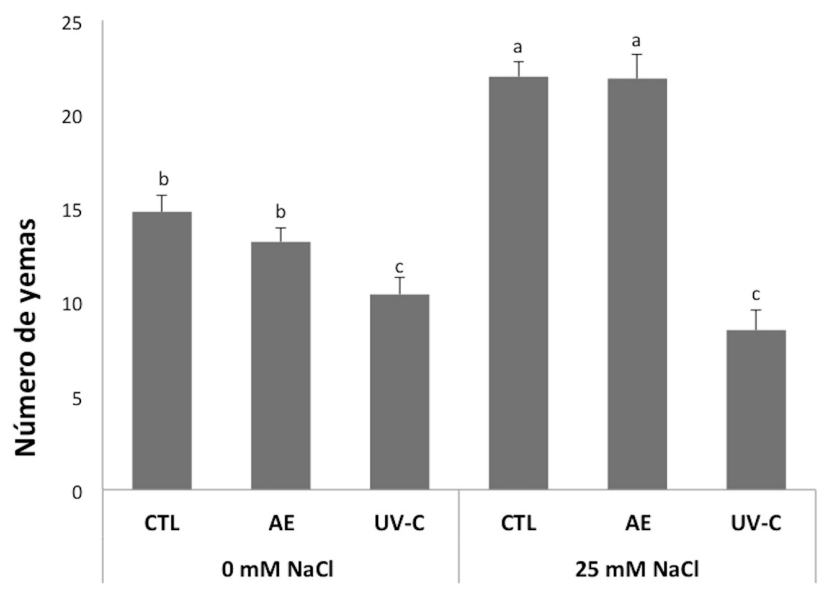

Figura 1. Número de yemas activadas. Se muestra el efecto del estrés salino sobre el crecimiento de plantas de orégano cultivadas in vitro bajo distintos tipos de iluminación. Las barras representan el valor promedio de cada aspecto evaluado y se muestran las barras de error. Las letras diferentes sobre las barras indican diferencias significativas $(a=0.05)$.

Figure 1. Activated buds. The effect of saline stress on oregano plants grown in vitro under different light conditions is shown. Bars represent mean value for each aspect evaluated, showing the standard error. Different letters on the bars indicate significant differences $(a=0.05)$.

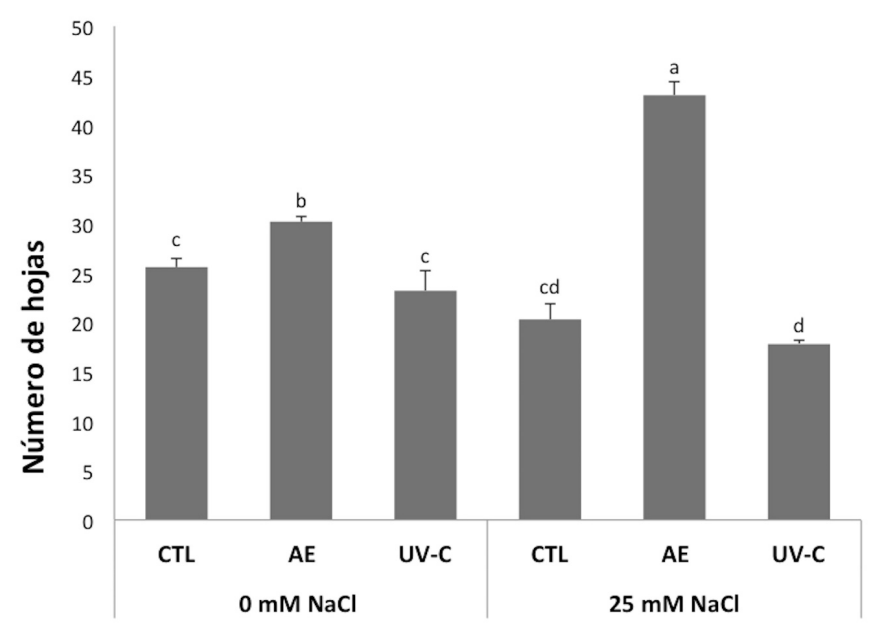

Figura 2. Altura de las plantas. Se muestra el efecto del estrés salino sobre el crecimiento de plantas de orégano cultivadas in vitro bajo distintos tipos de iluminación. Las barras representan el valor promedio de cada aspecto evaluado y se muestran las barras de error. Las letras diferentes sobre las barras indican diferencias significativas $(a=0.05)$.

Figure 2. Plant height. The effect of saline stress on oregano plants grown in vitro under different light conditions is shown. Bars represent mean value for each aspect evaluated, showing the standard error. Different letters on the bars indicate significant differences $(a=0.05)$.

(MSBC). El carbono asignado para la síntesis de MSBC puede entrar a diversas vías como por ejemplo la del ácido shikímico o del mevalónico para la síntesis de fenilpropanoides, taninos hidrolizables y terpenoides (Valifard et al., 2014). La cantidad de carbono disponible que será destinado a la síntesis de MSBC como fenoles y terpenoides está regulada parcialmente por los genes, pero también está influenciado por el ambiente. Por esta razón es importante determinar los 


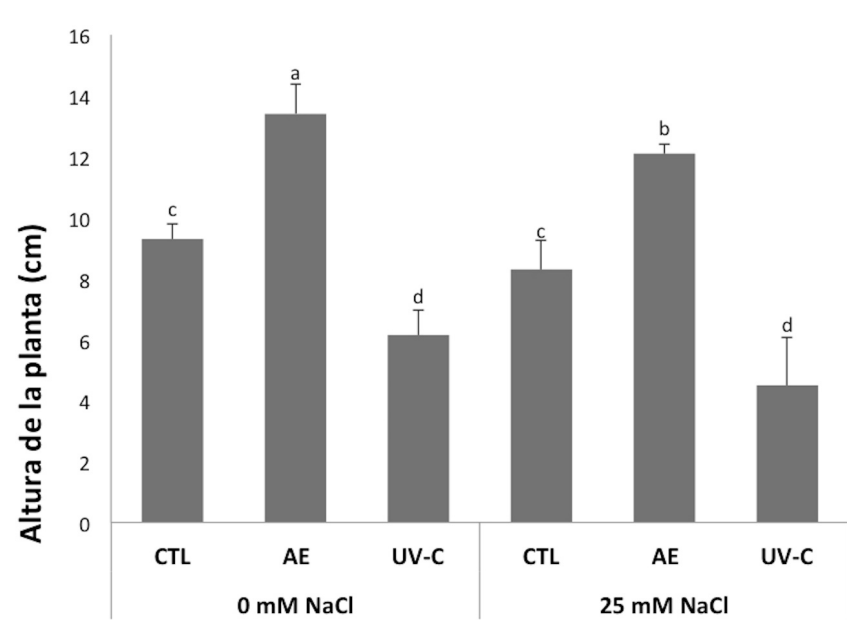

Figura 3. Número de hojas. Se muestra el efecto del estrés salino sobre el crecimiento de plantas de orégano cultivadas in vitro bajo distintos tipos de iluminación. Las barras representan el valor promedio de cada aspecto evaluado y se muestran las barras de error. Las letras diferentes sobre las barras indican diferencias significativas $(a=0.05)$.

Figure 3. Number of leaves. The effect of saline stress on oregano plants grown in vitro under different light conditions is shown. Bars represent mean value for each aspect evaluated, showing the standard error. Different letters on the bars indicate significant differences $(a=0.05)$.

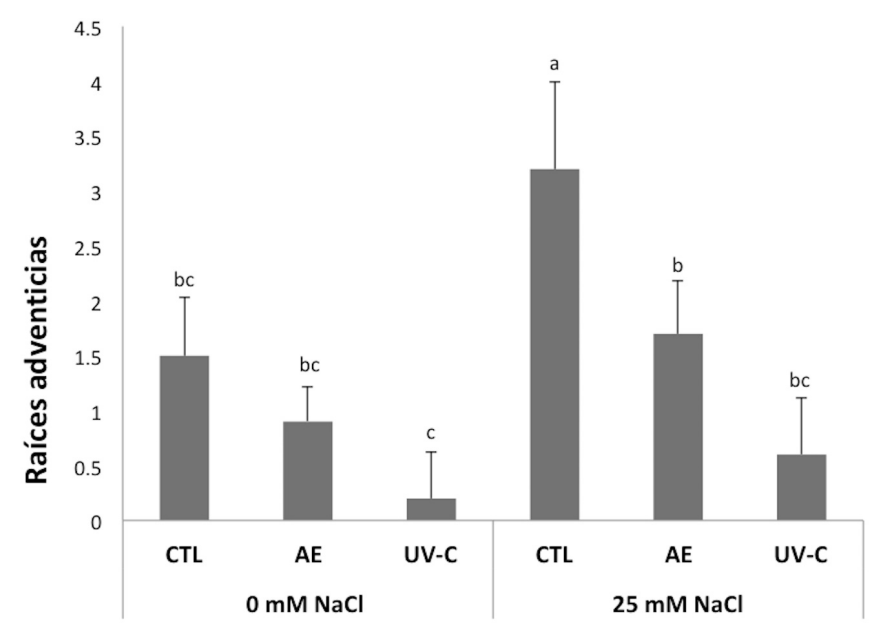

Figura 4. Número de raíces adventicias. Se muestra el efecto del estrés salino sobre el crecimiento de plantas de orégano cultivadas in vitro bajo distintos tipos de iluminación. Las barras representan el valor promedio de cada aspecto evaluado y se muestran las barras de error. Las letras diferentes sobre las barras indican diferencias significativas $(a=0.05)$.

Figure 4. Number of adventitious roots. The effect of saline stress on oregano plants grown in vitro under different light conditions is shown. Bars represent mean value for each aspect evaluated, showing the standard error. Different letters on the bars indicate significant differences $(a=0.05)$.

niveles de estos metabolitos cuando se realizan investigaciones con plantas bajo estrés (Selmar, 2008; Valifard et al., 2014). En este estudio se observó que los tratamientos $A E$ y UV-C causaron un incremento en los fenoles totales comparado con CTL, sin embargo, solo en el tratamiento $\mathrm{NaCl} /$ CTL se observó efecto por la salinidad ya que incrementó la cantidad de los fenoles totales. Este resultado concuerda con lo reportado por varios autores sobre el incremento de los niveles de los compuestos fenólicos totales en plantas sometidas a condiciones de estrés salino (Sytar et al., 2018; Isah, 2019), sin embargo, Selmar indicó en el año 2008 que este incremento en los metabolitos secundarios puede no ser la única respuesta, sino que también se debe tomar en cuenta que, por efecto del estrés, muchas plantas sufren una reducción en su crecimiento. Como consecuencia, este incremento en los metabolitos secundarios comparado con plantas no estresadas podría simplemente deberse a que en las plantas estresadas se encuentra menos biomasa que en las plantas no tratadas. Recomienda llevar a cabo estudios sobre la cantidad de biomasa para poder descartar este supuesto (Selmar, 2008).

En el caso de los tratamientos $\mathrm{NaCl} / \mathrm{AE}$ y $\mathrm{NaCl} / \mathrm{UV}-\mathrm{C}$ no se observó un aumento en los fenoles totales, comparado con AE y UV-C, respectivamente (Tabla 2).

\section{Flavonoides totales}

El estrés salino genera estrés osmótico en las plantas, debido a la sobreproducción de ERO a través de vías como la fotorrespiración, fotosíntesis y la respiración mitocondrial (Sharma et al., 2013). Como respuesta a este estrés osmótico, las plantas cuentan con distintas vías de neutralización de las ERO, tanto enzimáticas como no enzimáticas. Para adaptarse, plantas en condiciones de estrés salino tienden a acumular solutos como prolina, proteínas y carbohidratos solubles y antioxidantes como ácido ascórbico, fenoles y flavonoides, causando una disminución del potencial osmótico en el citoplasma, lo cual permite la absorción de agua (Sarker y Oba, 2018). El contenido de flavonoides totales mostró que el tratamiento $A E$ no indujo un cambio significativo en su contenido, comparado con las plantas control (CTL). Sin embargo, en los tratamientos $\mathrm{NaCl} / \mathrm{CTL}$ y $\mathrm{NaCl} / \mathrm{UV}-\mathrm{C}$ se observó el efecto de la salinidad sobre el contenido de los flavonoides. En general, el estrés por luz ultravioleta provocó un incremento en los flavonoides totales (UV-C y NaCl/UV-C), efecto que no se observó para $\mathrm{NaCl} / \mathrm{AE}$, ya que este último tratamiento no causó un incremento en los flavonoides, resultado que también se observó en los fenoles totales donde la menor cantidad de fenoles totales fue en el tratamiento $\mathrm{NaCl} / \mathrm{AE}$ (Tabla 2). Estos resultados indican que el efecto que tiene la luz UV-C sobre la producción de flavonoides es mayor que aquel efecto del $\mathrm{NaCl} / \mathrm{CTL}$. Los resultados concuerdan con la literatura donde varios autores reportan un aumento en el contenido de flavonoides totales en plantas sometidas a condiciones de estrés salino (Ahmed et al., 2012; Mzabri et al., 2017; Zhou et al., 2018).

\section{Capacidad antioxidante}

Como se mencionó anteriormente, cuando se someten a las plantas a condiciones de estrés salino, o en general el estrés abiótico, resulta en un desbalance iónico en las células. Este desbalance causa la generación de ERO y como respuesta los diversos mecanismos antioxidantes. Existen muchas técnicas para medir la capacidad antioxidante, cada una con sus limitaciones y virtudes. Dentro de las más utiliza- 
Tabla 2. Parámetros fitoquímicos de L. graveolens.

Table 2. Phytochemical parameters of L. graveolens.

\begin{tabular}{|c|c|c|c|c|}
\hline Tratamiento & $\begin{array}{c}\text { Fenoles } \\
\text { totales } \\
\text { (mg EAG/gps) }^{1}\end{array}$ & $\begin{array}{l}\text { Flavonoides } \\
\text { totales } \\
\text { (mg EQ/gps) }^{2}\end{array}$ & $\begin{array}{c}\text { Capacidad } \\
\text { antioxidante DPPH } \\
\text { (CAET mM/gps) }^{3}\end{array}$ & $\begin{array}{c}\text { Capacidad } \\
\text { antioxidante ABTS } \\
\text { (CAET mM/gps) }^{3}\end{array}$ \\
\hline CTL & $6.19 \pm 0.25^{\mathrm{a}}$ & $0.86 \pm 0.10^{a}$ & $0.52 \pm 0.011^{a}$ & $87.45 \pm 0.28^{a}$ \\
\hline $\mathrm{AE}$ & $6.61 \pm 0.20^{\mathrm{a}}$ & $0.77 \pm 0.16^{a}$ & $1.76 \pm 0.04^{b}$ & $25.74 \pm 1.98^{b}$ \\
\hline UV-C & $9.52 \pm 0.27^{b}$ & $3.65 \pm 0.10^{b}$ & $0.34 \pm 0.00^{c}$ & $104.52 \pm 1.19^{c}$ \\
\hline $\mathrm{NaCl} / \mathrm{CTL}$ & $8.28 \pm 0.03^{c}$ & $1.34 \pm 0.16^{c}$ & $1.53 \pm 0.04^{d}$ & $199.1 \pm 1.45^{d}$ \\
\hline $\mathrm{NaCl} / \mathrm{AE}$ & $4.95 \pm 0.28^{d}$ & $0.43 \pm 0.07^{d}$ & $2.01 \pm 0.02^{\mathrm{e}}$ & $337.39 \pm 0.34^{\mathrm{e}}$ \\
\hline $\mathrm{NaCl} / \mathrm{UV}-\mathrm{C}$ & $7.83 \pm 0.46^{c}$ & $4.01 \pm 0.13^{e}$ & $0.74 \pm 0.04^{f}$ & $100.42 \pm 0.93^{f}$ \\
\hline
\end{tabular}

Cada valor es la media de tres repeticiones \pm desviación estándar. Valores seguidos por la misma letra no son diferentes de acuerdo con la comparación múltiple de Tukey $(p=0.05)$.

1-miligramos equivalentes de ácido gálico/gramo peso seco

2-miligramos equivalentes de quercetina/gramo peso seco

3-capacidad antioxidante equivalentes de trolox milimolar/gramo peso seco

das se encuentran el método que usa el radical DPPH y el que usa al ABTS. El DPPH es considerado un radical libre y estable de coloración violeta. En solución metanólica absorbe a una longitud de onda de alrededor de $517 \mathrm{~nm}$ y al mezclarse esta solución con una sustancia capaz de donar un átomo de hidrógeno se genera la forma reducida, perdiendo el color violeta (Kedare y Singh, 2011). El ABTS es un cromóforo de coloración azul turquesa con una absorción máxima a 734 $\mathrm{nm}$. En la presencia de antioxidantes, disminuye la coloración de este radical sea por donación de un electrón o por atrapamiento del radical a través de la donación de átomos de hidrógeno (Zhong y Shahidi, 2015).

Los resultados obtenidos en el ensayo con DPPH mostraron diferencias significativas entre todos los tratamientos y entre los tratamientos y el control. El tratamiento que mayor capacidad antioxidante mostró fue $\mathrm{NaCl} / \mathrm{AE}$, mientras que UV-C mostró la menor capacidad. Sin embargo, las tres condiciones de salinidad ( $\mathrm{NaCl} / \mathrm{CTL}, \mathrm{NaCl} / \mathrm{AE}$ y $\mathrm{NaCl} /$ UV-C) mostraron un aumento en la capacidad antioxidante, comparado con las condiciones sin sal. Los tratamientos con luz UV-C causaron una disminución en la capacidad antioxidante (Tabla 2). Los resultados de la capacidad antioxidante con ABTS también mostraron diferencias significativas entre todos los tratamientos. El tratamiento AE mostró la menor capacidad antioxidante, incluso menor que el control, seguido por el tratamiento $\mathrm{NaCl} / \mathrm{UV}-\mathrm{C}$ y finalmente la combinación $\mathrm{NaCl} / \mathrm{AE}$ fue la que mayor capacidad antioxidante mostró (Tabla 2). En los tratamientos $\mathrm{NaCl} / \mathrm{CTL}$ y $\mathrm{NaCl} / \mathrm{AE}$ se observa claramente el efecto de la salinidad, aumentando la capacidad antioxidante con respecto a CTL y $A E$, respectivamente. El tratamiento UV-C mostró un aumento en la capacidad antioxidante con respecto a CTL, sin embargo, la salinidad no provocó un aumento en la capacidad antioxidante si se compara UV-C con NaCl/UV-C. Los resultados generales de la capacidad antioxidante (DPPH y ABTS) mostraron que $\mathrm{NaCl} /$ $A E$ fue el tratamiento que más disparó esta capacidad y que en general la salinidad provocó un aumento en la capacidad antioxidante. Estos resultados concuerdan con la literatura donde se reportó un aumento en la capacidad antioxidante por efecto del estrés salino (Ahmed et al., 2012). No se observó una clara relación de la capacidad antioxidante con los compuestos fenólicos totales. Esto no concuerda con lo reportado en la literatura ya que se sabe que los compuestos fenólicos tienen una alta correlación con la capacidad antioxidante, gracias a su facilidad de donar átomos hidrógeno de los grupos hidroxilo (Pereira et al., 2009; Bagues et al., 2019). Esto nos hace pensar que la capacidad antioxidante observada en este estudio no proviene solo de los fenoles totales, sino que también aportaron antioxidantes de otra naturaleza. Algunos de los fenoles reportados en L. graveolens son timol, carvacrol, quercetina, O-hexosido, escutelarina etc. (González-Trujano et al., 2017). La identidad de los fenoles y flavonoides que se extrajeron en este estudio y su relación directa con la capacidad antioxidante no fueron determinados en este estudio, sin embargo, actualmente se está trabajando en esta parte.

El ensayo con ABTS mostró mayor capacidad antioxidante que el ensayo DPPH y esto se debe a que cada método de análisis tiene diferentes mecanismos de acción. El ensayo $A B T S$ se basa en la generación del radical $A B T S^{+}$que es aplicable a los sistemas antioxidantes tanto hidrofílicos como lipofílicos mientras que el ensayo DPPH usa un radical disuelto en medios orgánicos y, por lo tanto, se aplica mayoritariamente a sistemas hidrofóbicos (Rivero-Pérez et al., 2007; San Miguel-Chávez, 2017).

\section{CONCLUSIÓN}

En general se observó que el tratamiento con luz UV-C causó un efecto negativo en el crecimiento y desarrollo de las plantas. Los tratamientos $\mathrm{NaCl} / \mathrm{CTL}$ y $\mathrm{NaCl} / \mathrm{AE}$ causaron el mayor número de yemas activas, mientras que $\mathrm{AE}$ y $\mathrm{NaCl} / \mathrm{AE}$ causaron el mayor crecimiento de las partes aéreas. Además, $\mathrm{NaCl} / \mathrm{AE}$ fue el tratamiento que resultó en el mayor número de hojas. De todas las condiciones estresantes, la luz UV-C generó la mayor cantidad de compuestos fenólicos totales y la combinación $\mathrm{NaCl} / \mathrm{AE}$ fue la que menor producción de fenoles causó. Esta misma combinación estresante causó la menor cantidad de flavonoides, siendo el tratamiento $\mathrm{NaCl} /$ 
UV-C el que mostró la mayor cantidad de flavonoides totales. En general, la salinidad causó un incremento en la capacidad antioxidante (DPPH y ABTS) siendo el tratamiento $\mathrm{NaCl} / \mathrm{AE}$ el que mayor número de capacidad antioxidante mostró, tanto para DPPH como para ABTS. No se observó una correlación entre fenoles y capacidad antioxidante como generalmente suele suceder, ya que la condición estresante que resultó en la mayor capacidad antioxidante no coincide con la que mostró la mayor cantidad de fenoles o flavonoides totales.

\section{REFERENCIAS}

Ahmed A., Ahmed M., Al- Sayed H., Smetanska I. (2012). Effect of Drought and Salinity Stress on Total Phenolic, Flavonoids and Flavonols Contents and Antioxidant Activity in in vitro Sprout cultures of Garden cress (Lepidium sativum). Journal of Applied Sciences Research 8: 3934-3942.

Arif M., Islam M., Robin A. (2019). Salinity Stress Alters Root Morphology and Root Hair Traits in Brassica napus. Plants 8: 192.

Bagues M., Hafsi C., Yahia Y., Souli I., Boussora F., Nagaz K. (2019). Modulation of Photosynthesis, Phenolic Contents, Antioxidant Activities, and Grain Yield of Two Barley Accessions Grown under Deficit Irrigation with Saline Water in an Arid Area of Tunisia. Polish Journal of Environmental Studies 5: 3071-3080.

Brand-Williams W., Cuvelier M., Berset C. (1995). Use of a Free Radical Method to Evaluate Antioxidant Activity. Lebensmittel-Wissenschaft + Technologie 28: 25-30.

Chang C., Yang M., Wen H., Chern J. (2002). Estimation of total flavonoid content in propolis by two complementary colorimetric methods. Journal of Food and Drug Analysis 10: $178-82$.

Gill S., Tuteja N. (2010). Reactive oxygen species and antioxidant machinery in abiotic stress tolerance in crop plants. Plant Physiology and Biochemistry 48: 909-930.

González-Trujano M., Hernández-Sánchez L., Muñoz Ocotero V., Dorazco-González A., Guevara Fefer P., Aguirre-Hernández E. (2017). Pharmacological Evaluation of the anxiolytic-like Effects of Lippia graveolens and Bioactive Compounds. Pharmaceutical Biology 55: 1569-1576.

Herrera-Rodríguez S., López-Rivera R., García-Márquez E., Estarrón-Espinoza M., Espinosa-Andrews H. (2019). Mexican Oregano (Lippia graveolens) Essential oil-in-water Emulsions: Impact of Emulsifier type on the Antifungical Activity of Candida albicans. Food Science and Biotechnology 28: 441 448.

Isah T. (2019). Stress and Defense Responses in Plant Secondary Metabolites Production. Biological Research 52:39.

Isayenkov S., Maathuis F. (2019). Plant Salinity Stress: Many Unanswered Questions Remain. Frontiers in Plant Science 10:80.

Kedare S., Singh R. (2011). Genesis and Development of DPPH method of Antioxidant Assay. Journal of Food Science and Technology 48: 412-422.

Keyvan S. (2010). The Effects of Drought Stress on Yield, Relative Water Content, Proline, Soluble carbohydrates and Chlorophyll of Bread Wheat Cultivars. Journal of Animal and Plant Sciences 8: 1051-1060.

Kotagiri D., Kolluru V. (2017). Effect of Salinity Stress on the Morphology \& Physiology of Five Different Coleus Species.
Biomedical \& Pharmacology Journal 10: 1639-1649.

López-Villafranco M., Aguilar-Contreras A., Aguilar-Rodríguez S., Xolalpa-Molina S. (2017). Las Verbenaceae Empleadas como Recurso Herbolario en México: Una Revisión EtnobotánicaMédica. Polibotánica 44: 195-216.

Martínez-Hernández R., Villa-Castorena M., Catalán-Valencia E., Inzunza-Ibarra M. (2015). Production of Oregano (Lippia graveolens Kunth) Seedlings from Seeds in Nursery for transplanting. Revista Chapingo Serie Ciencias Forestales y del Ambiente 23: 61-73.

Mata-González R., Meléndez-González R. (2005). Growth Characteristics of Mexican Oregano (Lippia Berlandieri Schauer) Under Salt Stress. The Southwestern Naturalist 50: 1-6.

Murashige T., Skoog F. (1962). A revised medium for rapid growth and bioassays with tobacco tissue cultures. Physiology Plantarum 15: 473-497.

Mzabri I., Legsayer M., Kouddane N., Boukroute A., Berrichi A. (2017). Salt Stress Effects on Some Morphological, Physiological and Biochemical Parameters of Saffron Plant (Crocus sativus L.) in Eastern Morocco. Journal of Materials and Environmental Sciences 8: 4894-4901.

Pascual M., Slowing K., Carretero E., Sánchez Mata D., Villar A. (2001). Lippia: Traditional Uses, Chemistry and Pharmacology: A Review. Journal of Ethnopharmacology 76: 201-214.

Pereira D., Valentão P., Pereira J., Andrade P. (2009). Phenolics: From Chemistry to Biology. Molecules 14: 2202-2211.

Rahdari P., Hoseini S. (2012). Drought stress: A Review. International Journal of Agronomy and Plant Production 3: 443-336.

Rana R., Rehman S., Ahmed J., Bilal M. (2013). A Comprehensive Overview of Recent Advances in Drought Stress Tolerance Research in Wheat (Triticum aestivum L.) Asian Journal of Agriculture and Biology 1: 29-37.

Razzaq A., Ali A., Bin Safdar L., Zafar M., Rui Y., Shakeel A., Shaukat A., Ashraf M., Gong W., Yuan Y. (2019). Salt Stress Induces Physiochemical Alterations in Rice Grain Composition and Quality. Journal of Food Science 0: 1-7.

Re R., Pellegrini N., Proteggente A., Pannala A., Yang M., RiceEvans C. (1999). Antioxidant Activity Applying an Improved ABTS Radical Cation Decolorization Assay. Free Radical Biology and Medicine 26: 1231-1237.

Rivera G., Bocanegra-García V., Monge A. (2010). Traditional Plants as Source of Fundamental Foods: A Review. CyTAJournal of Food 8: 159-167.

Rivero-Pérez M., Muñiz P., González-Sanjosé M. (2007). Antioxidant Profile of Red Wines Evaluated by Total Antioxidant Capacity, Scavenger Activity, and Biomarkers of Oxidative Stress Methodologies. Journal of Agricultural and Food Chemistry 55: 5476-5483.

San Miguel-Chávez R. (2017). Phenolic Antioxidant Capacity: A Review of the State of the Art. En: Phenolic CompoundsBiological Activity. Soto-Hernández M., Palma-Tenango M., García-Mateos M. (ed.), p 59-74.

Sarker U., Islam M., Oba S. (2018). Salinity Stress Accelerates Nutrients, Dietary Fiber, Minerals, Phytochemicals and Antioxidant Activity in Amaranthus tricolor leaves. PLoS ONE 13: e0206388.

Sarker U., Oba S. (2019). Salinity Stress Enhances Color Parameters, Bioactive Leaf Pigments, Vitamins, Polyphenols, 
Flavonoids and Antioxidant Activity in Selected Amaranthus Leafy Vegetables. Journal of the Science of Food and Agriculture 99: 2275-2284.

Selmar D. (2008). Potential of Salt and Drought Stress to Increase Pharmaceutical Significant Secondary Compounds in Plants. Landbauforschung-vTI Agriculture and Forestry Research 1/2: 139-144.

Sharif I., Aleem S., Farooq J., Rizwan M., Younas A., Sarwar S., Chohan S. (2019). Salinity Stress in Cotton: Effects, Mechanism of Tolerance and its Management Strategies. Physiology and Molecular Biology of Plants 25:807-820.

Sharma I., Ching E., Saini S., Bhardwaj R., Pati P. (2013). Exogenous application of brassinosteroid offers tolerance to salinity by altering stress responses in rice variety Pusa Basmati-1. Plant Physiology and Biochemistry 69. pp 17-26.

Sharma A., Shahzad B., Rehman A., Bhardwaj R., Landi M., Zheng B. (2019). Response of Phenylpropanoid Pathway and the Role of Polyphenols in Plants under Abiotic Stress. Molecules 24: 2452.

Singleton V., Rossi J. (1965). Colorimetry of Total Phenolics with Phosphomolyb-diphosphotungstic Acid Reagents. American Journal of Enology and Viticulture 16: 144- 158.
Sytar O., Barki S., Zivcak M., Brestic M. (2018). The involvement of different secondary metabolites in salinity tolerance of crops. En: Salinity responses and tolerance in plants, vol. 2. Kumat V. (ed.), pp 21-48. Berlin: Springer International Publishing AG, part of Springer Nature.

Valifard M., Mohsenzadeh S., Kholdebarin B., Rowsan V. (2014). Effects of Salt Stress on Volatile Compounds, Total Phenolic Content and Antioxidant Activities of Salvia mirzayanii. South African Journal of Botany 93: 92-97.

Zhong Y., Shahidi F. (2012). Methods for the Assessment of Antioxidant Activity in Foods. En: Handbook of Antioxidants for Food Preservation. Shahidi F. (ed.), pp 287-333. Woodhead Publishing.

Zlatev Z., Lidon C. (2012). An Overview on Drought Induced Changes in Plant Growth, Water Relations and Photosynthesis. Emirates Journal of Food and Agriculture 24: 57-72.

Zhou Y., Tang N., Huang L., Zhao Y., Tang X., Wang K. (2018). Effects of Salt Stress on Plant Growth, Antioxidant Capacity, Glandular Trichome Density, and Volatile Exudates of Schizonepeta tenuifolia Briq. International Journal of Molecular Sciences 19: 252. 